\title{
CARACTERIZAÇÃO DO FATOR CAPACIDADE DE FÓSFORO EM SOLOS DE ENTRE RÍOS, ARGENTINA ${ }^{(1)}$
}

\author{
N. G. BOSCHETTI A. ${ }^{(2)}$, C. E. QUINTERO G. ${ }^{(2)} \&$ R. A. BENAVIDEZ Q. ${ }^{(2)}$
}

\begin{abstract}
RESUMO
Trinta amostras de solos foram selecionadas com o objetivo de caracterizar o Fator Capacidade de Fósforo (FCP) e sua relação com propriedades de solos de Entre Ríos, Argentina. F oram estimadas i soter mas de adsorção e realizado um teste rápido de adsorção de $P$, denominado $P$-remanescente. As equações de Freundlich e Langmuir descreveram adequadamente o fenômeno de adsorção de P. A capacidade máxima de adsorção variou de 46 a $716 \mathrm{mg} \mathrm{kg}^{-1}$ de $P$ no solo, apresentando os solos analisados de muito baixo a médio FCP. A capacidade de adsorção de $P$ aumentou com a quantidade de argila e não foi afetada pelo teor de carbono orgânico do solo. O P-remanescente permitiu caracterizar os solos em relação ao fator capacidade e estimar sua capacidade máxima de adsorção de P.
\end{abstract}

Termos de indexação: fósforo, fator capacidade, argila, isotermas de adsorção, P-remanescente, carbono orgânico.

\section{SUMMARY: CHARACTERIZATION OF THE CAPACITY FACTOR OF PHOSPHORUS IN SOILS OF ENTRE RIOS, ARGENTINA}

Samples of 30 soils weretaken with the objective of characterizing the Capacity Factor of Phosphorus (CFP) and its relation with several properties of soils of Entre Ríos, Argentina. I sotherms of adsorption and a quick test of $P$ adsorption were estimated. Langmuir and Freundlich equations described the adsorption phenomenon satisfactorily. The maximum adsorption capacity varied between 46 and $716 \mathrm{mg} \mathrm{kg}^{-1}$ of $P$ in the soil, considering that the soils analyzed havefrom medi um to low CFP. Theadsorption capacity of $P$ increased with clay content and wasn't affected by the soil's organic carbon concentration. The quick adsorption test permi tted thecharacterization of thebuffer capacity of soils and theestimate of its maximum adsorption capacity of $P$.

Index terms: phosphorus, buffer capacity, clay, isotherms of adsorption, remaining-P, organic carbon.

\footnotetext{
(1) Trabal ho apresentado no XXV Congresso Brasileiro de Ciência do Solo. Recebido para publicação em março de 1996 e aprovado em janeiro de 1998.

(2) Professor do Departamento de Ciência da Terra, Universidade Nacional de Entre Ríos. Faculdade de Ciências Agropecuárias. CC24 Paraná ER (3100), Argentina. <ediaz@unl.edu.ar>.
} 


\section{INTRODUÇÃO}

A capacidade dos solos em adsorver $P$ influencia, marcadamente, a resposta das plantas à apl icação de fertilizantes e à calibração do $\mathrm{P}$ disponível do solo. Portanto, o conhecimento da capacidade tampão ou fator capacidade de $\mathrm{P}$ do solo ou de alguma propriedade do solo a ele correlacionada poderia melhorar a interpreta-ção de análises dos solos e a estimativa do requerimento de fósforo para os cultivos (Freire et al., 1979; Cajuste et al., 1992; Moughli et al., 1993; Ron et al., 1995).

Para determinar a capacidade de adsorção de P dos solos, duas aproximações têm sido, normal mente, utilizadas. Freqüentemente, equilibram-se amostras de solo com soluções diluídas de fosfato, determinando-se a quantidade de $\mathrm{P}$ adsorvida (OIsen \& Watanabe, 1957; Ozanne\& Shaw, 1968). I sotermas deadsorção são ajustadas aos resultados obtidos desse equilíbrio. Dadas as dificul dades desse procedimento, estimativas mais simples têm sido propostas, com o objetivo de serem adotadas nos laboratórios de rotina (Alvarez V., 1982; Muniz et al., 1985). Para a segunda alternativa, identificam-se propriedades de solo que têm mostrado estreita relação com a capacidade de adsorção de $\mathrm{P}$ dos solos. Tais propriedades podem incluir a textura, pH , C orgânico, F eeAl reativos, etc (López Camelo \& Heredia, 1989; Dear et al., 1992).
Na Argentina, foram realizadas determinações do fator capacidade de $\mathrm{P}$ em regiões do Pampa Úmido (López Camelo \& Heredia, 1989) do Pampa Central (Buschiazzo et al., 1990) e outras, a nível nacional, incluindo alguns solos de Entre Ríos (Bolaño, 1984; López Camelo et al., 1984, Mendoza, 1986).

O objetivo deste trabalho foi caracterizar o fator capacidade de $\mathrm{P}$ de diferentes solos do Estado deE ntre Ríos, Argentina, bem como avaliar sua relação com algumas propriedades desses solos.

\section{MATERIAL E MÉTODOS}

\section{Solos e propriedades avaliadas}

Foram coletadas 27 amostras superficiais e três subsuperficiais de solos do Estado de Entre Ríos, que representam os mais importantes tipos de solos da região (sete Molisols, noveVertisols, seteAlfisols, três Entisols, um I nceptisol etrês de horizontes B textural deVertisols). As amostras foram secas ao ar e passadas por peneira de $1 \mathrm{~mm}$. As principais propriedades físicas e químicas dos sol os encontram-se no quadro 1.

Quadro 1. Classificação, propriedades físicas e químicas e resultados dos índices que refletem o fator capacidade de $\mathbf{P}$ dos solos estudados

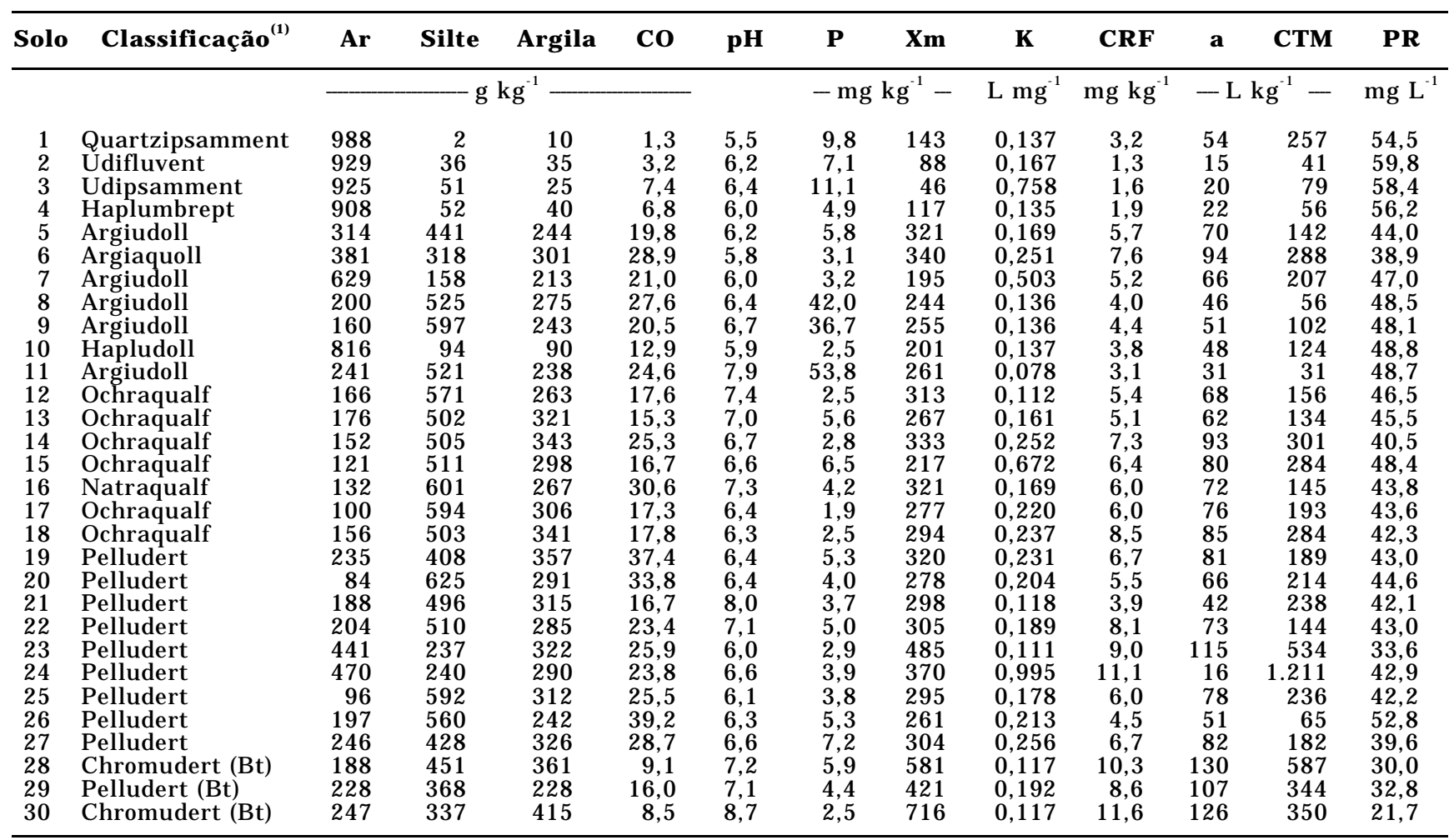

${ }^{(1)}$ Soil Taxonomy. Ar: Areia. CO: Carbono orgânico, Walkley-Black. pH em água, relação 1:2,5. P: Fósforo disponível, Bray-Kurtz 1. Xm: Capacidade máxima de adsorção de P. K: Constante relacionada com a energia de adsorção de $P$. a: Constante de Freundlich. CRF : Capacidade reguladora de fósforo. CTM: Capacidade tampão máxima calculada com os valores de Xm e K da primeira região. PR: Fósforo remanescente. 


\section{Índices do fator capacidade de fósforo (FCP)}

- Í ndice de pontoúnico ou testerápido: foi realizada a determinação de fósforo-remanescente (PR), que expressa a concentração de $P$ na sol ução de equilíbrio depois de agitar uma solução de $60 \mathrm{mg} \mathrm{L}^{-1}$ de $\mathrm{P}$ em $\mathrm{CaCl}_{2} 0,01 \mathrm{~mol} \mathrm{~L}^{-1}$, por uma hora, em uma relação de solo solução 1:10, a 21ํC (Alvarez V., 1982).

- I sotermas de adsorção: foi utilizada uma relação solo-solução de 1:10, agitação vertical por 24 horas, à temperatura de $21^{\circ} \mathrm{C}$. Como fonte de $\mathrm{P}$, utilizou-se $\mathrm{KH}_{2} \mathrm{PO}_{4}$ em uma solução de $\mathrm{CaCl}_{2} 0,01 \mathrm{~mol} \mathrm{~L}^{-1}$, com duas gotas de tolueno. Para selecionar as doses aplicadas, foram levadas em conta as sugestões de Alvarez V. \& Fonseca (1990) e testes preliminares. Os solos foram classificados em quatro grupos em função de seu PR; foram utilizadas onze doses de P (Quadro 2).

Para descrever o fenômeno de adsorção de $P$, ajustou-sea equação de Langmuir (Equação 1) (Olsen \& Watanabe, 1957), estimando-se suas constantes correspondentes à segunda região de adsorção, em que se cumprem as pressuposições para o uso adequado dessa isoterma (Alvarez V., 1982; Quintero et al., 1996). Ajustou-se, também, a equação de Freundlich (Equação 2).

$$
\begin{gathered}
q=\frac{K X m C}{1+K C}, \\
q=a c^{b},
\end{gathered}
$$

em que

$\mathrm{q}=\mathrm{F}$ ósforo adsorvido ( $\mathrm{mg} \mathrm{kg}^{-1}$ de $\mathrm{P}$ no solo). $\mathrm{C}=$ Concentração da solução de equilíbrio $\left(\mathrm{mg} \mathrm{L}^{-1} \mathrm{de} P\right.$ em solução). $\mathbf{K}=$ Constante relacionada com a energia de adsorção de fósforo $\left(\mathrm{L} \mathrm{mg}^{-1}\right)$. $\mathbf{X m}=$ Capacidade máxima de adsor ção de fósforo ( $\mathrm{mg} \mathrm{kg}^{-1}$ de P no solo). a e $\underline{\mathbf{b}}$ : Constantes de F reundlich.

Utilizaram-se como índices do fator capacidade as constantes K e Xm de Langmuir e a de Freundlich. Além disso, foi estimada a Capacidade Tampão Máxima (CTM) como a declividade máxima da isoterma de Langmuir, quando a concentração da solução de equilíbrio tende a zero (Holford \& Mattingly, 1976). Foi também cal culada a Capacidade Reguladora de Fosfatos (CRF), definida como a

Quadro 2. Doses de fósforo adicionadas aos di ferentes grupos de solos, de acordo com o P-remanescente (PR), para determinação das isotermas de adsorção

\begin{tabular}{lrrrrrrrrrrr}
\hline \multicolumn{10}{c}{ PR } & \multicolumn{10}{c}{ Doses de P } \\
\hline \multicolumn{10}{c}{ mg L $^{-1}$} \\
\hline 30 & 0 & 5,0 & 9,0 & 15 & 24 & 36 & 50 & 60 & 70 & 80 & 100 \\
$30-45$ & 0 & 5,0 & 9,0 & 15 & 20 & 30 & 40 & 50 & 60 & 70 & 80 \\
$45-55$ & 0 & 3,5 & 7,0 & 9 & 15 & 20 & 24 & 30 & 40 & 50 & 60 \\
$>55$ & 0 & 2,0 & 3,5 & 5 & 7 & 9 & 12 & 15 & 20 & 24 & 30 \\
\hline
\end{tabular}

quantidade de $\mathrm{P}$ adsorvido pelo solo quando a concentração de equilíbrio aumenta de 0,25 para $0,35 \mathrm{mg} \mathrm{L}^{-1}$ de $\mathrm{P}$ (Ozanne \& Shaw, 1968).

\section{RESULTADOS E DISCUSSÃO}

\section{Caracterização do fator capacidade de fósforo}

Tanto para a equação de Langmuir como para a de Freundlich, conseguiram-se ajustes satisfatórios, com val ores de $\mathrm{R}^{2}$ acima de 0,94 , o que indica a adequada descrição do fenômeno de adsorção de P pel os solos. Em todos os sol os, observaram-seas três regiões de adsorção da forma linear de Langmuir, propostas por Muljadi et al. (1966). Os resultados obtidos para os distintos solos são apresentados no quadro 1.

Os valores de $\mathbf{X m}$ encontrados para Molisols, Vertisols e Alfisols são superiores aos apresentados por López Camel o et al. (1984); Mendoza (1986); López Camelo \& Heredia (1989) e Buschiazzo et al. (1990). Possivel mente, esses autores utilizaram concentrações de fósforo menores e estimaram essa constante a partir da primeira região de adsorção, onde Xm é menor (Alvarez, V., 1982; Quintero et al., 1996). No caso dos Entisols e I nceptisols, os val ores observados são similares aos encontrados por López Camelo et al. (1984) mas inferiores aos propostos por Buschiazzo et al. (1990), provavel mente em virtude da utilização de materiais derivados de cinzas vulcânicas por esses autores.

A equação de Freundlich comportou-se satisfatoriamente em todas as ampl itudes de concentrações deP utilizadas. Apesar deser uma isoterma empírica, sua constante a correlacionou-se significativamente com o valor de $\mathbf{X m}$ dos solos estudados. Todos os índices estimados (PR, Xm, a, CRF, CTM) correlacionaram-se significativamente entre si (Quadro 3).

Em trabal hos com Latossol os, tem-se observado que o PR apresenta estreita correlação com Xm, sendo, portanto, um bom indicador do fator capacidade de $\mathrm{P}$ do solo, por expressar a natureza e a extensão da superfície adsorvente de $\mathrm{P}$ e ser de fácil determinação em laboratório (Silva, 1990). A análise conjunta de val ores dePR eXm de Latossol os do Brasil (AlvarezV., \& Fonseca, 1990; Silva \& Braga, 1993) e de Andosols do México (Alvarez V., 1982), bem como os obtidos neste trabalho, mostra excel ente ajuste (Figura 1). I sto reafirma a sensibilidade do PR como medida do fator capacidade de $\mathrm{P}$ do solo, mesmo quando solos tão diferentes são utilizados. A partir de medidas de PR desses solos, foi possível caracterizá-los quanto a essa medida do fator capacidade de P (Quadro 4).

Os horizontes superficiais dos solos argentinos anal isados pertencem às classes baixa e muito baixa de fator capacidade, indicando não haver sérios problemas de adsorção e de fixação de $\mathrm{P}$ e que doses de $P$ requeridas para obter el evada produção vegetal são rel ativamente baixas. As amostras dos horizontes Bt apresentam poder de adsorção médio que al cança 
Quadro 3. Coeficientes de correlação linear si mples entre propriedades dos solos e constantes das equações de Langmuir e de Freundlich

\begin{tabular}{|c|c|c|c|c|c|c|c|c|c|}
\hline Variável & pH & $\mathbf{P}$ & Argila & PR & $\mathbf{X m}$ & $\mathbf{K}$ & $\mathbf{a}$ & CRF & СТM \\
\hline $\mathrm{CO}$ & $-0,02$ & 0,09 & $0,51^{* *}$ & $-0,17$ & 0,17 & $-0,01$ & 0,18 & 0,22 & 0,01 \\
\hline $\mathrm{pH}$ & & 0,22 & $0,49^{* *}$ & $-0,51^{* *}$ & $0,58^{* *}$ & $-0,15$ & 0,27 & $0,38^{*}$ & 0,06 \\
\hline $\mathrm{P}$ & & & $-0,11$ & 0,25 & $-0,18$ & $-0,19$ & $-0,39^{*}$ & $-0,35^{*}$ & $-0,30$ \\
\hline Argila & & & & $-0,84^{* *}$ & $0,79^{* *}$ & $-0,09$ & $0,73^{* *}$ & $0,80^{* *}$ & $0,36^{*}$ \\
\hline PR & & & & & $-0,96^{* *}$ & 0,17 & $-0,84^{* *}$ & $-0,91^{* *}$ & $-0,47^{* *}$ \\
\hline$\underline{X m}$ & & & & & & $-0,22$ & $0,82^{* *}$ & $0,89^{* *}$ & $0,50^{* *}$ \\
\hline$\underline{\mathbf{K}}$ & & & & & & & 0,24 & 0,15 & $0,52^{* *}$ \\
\hline$\underline{\mathbf{a}}$ & & & & & & & & $0,98^{* *}$ & $0,85^{* *}$ \\
\hline$\overline{\mathrm{CRF}}$ & & & & & & & & & $0,76^{* *}$ \\
\hline
\end{tabular}

CO: Carbono orgânico. pH em água. P: Fósforo disponível. XXm: Capacidade Máxima de Adsorção de P. K: Cosntante Relacionada com a Energia de Adsorção de P. a: Constante de Freundlich. CRF: Capacidade Reguladora de Fósforo. CTM: Capacidade Tampão Máxima. PR: Fósforo remanescente.

$* p<0,05 ; * * p<0,01$

Quadro 4. Classes de solos quanto ao fator capacidade de P estimado a partir do P-remanescente (PR)

\begin{tabular}{rlc}
\hline \multicolumn{1}{c}{$\mathbf{P R}$} & Capacidade de adsorção de $\mathbf{P}$ & $\mathbf{X m}$ \\
\hline $\mathrm{mg} \mathrm{L}^{-1}$ & & $\mathrm{mg} \mathrm{kg}^{-1}$ \\
$0-10$ & Muito Alta & $>1.100$ \\
$10-20$ & Alta & $1.100-700$ \\
$20-30$ & Média & $700-500$ \\
$30-45$ & Baixa & $500-300$ \\
$45-60$ & Muito baixa & $<300$ \\
\hline
\end{tabular}

Xm: Capacidade Máxima de Adsorção de P.

um valor de $\mathbf{X m}$ de $716 \mathrm{mg} \mathrm{kg}^{-1}$, que demonstra que problemas defixação deP poderão aumentar nos solos erodidos.

\section{Relação de FCP com propriedades dos solos}

Como outros autores têm observado (Bolaño, 1984; López Camelo et al.,1984; Mendoza, 1986; López Camelo \& Heredia, 1989; Silva, 1990; Singh \& Gilkes, 1991; Moughli et al., 1993), a quantidadede argila éa principal responsável pela variação do fator capacidade de $\mathrm{P}$ dos solos. Esta propriedade correlaciona-se significativamente com todos os índices avaliados, à exceção da energia deadsorção (Quadro 3). Entretanto, deve-se levar em conta oti po deargila, dados os valores mais altos de adsorção encontrados nos Andosols por Alvarez V. (1982) emsol os apenas com $210 \mathrm{~g} \mathrm{~kg}^{-1}$ deargila.

Diversos autores verificaram relação direta entre o teor de carbono orgânico e a adsorçã̃o máxima de $\mathrm{P}$ (Bahia Filho, 1974; Bolaño, 1984; Hepper et al., 1995). Esses autores não esclareceram se essa correlação se deve a uma associação indireta entre o conteúdo de argila e carbono orgânico, como demonstraram Moughli et al. (1993). Nestetrabal ho, não se observou correlação linear simples significativa entre carbono

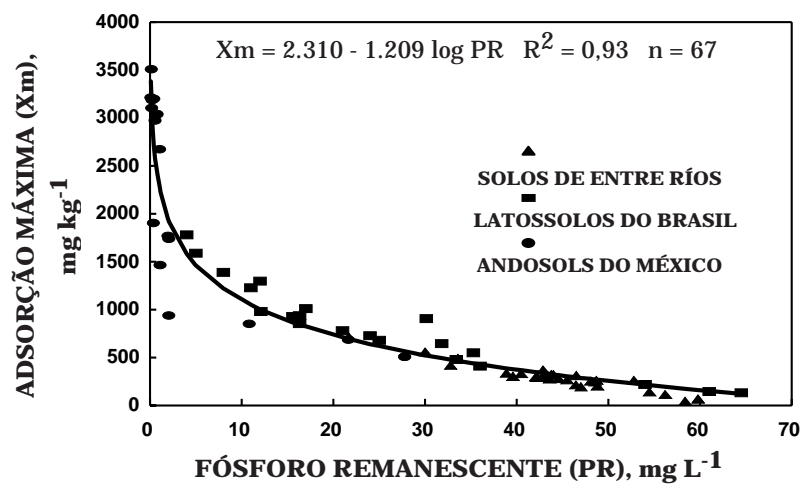

Figura 1. Regressão entre a capacidade máxima de adsorção de $\mathbf{P}$ e o fósforo remanescente. Os coeficientes são significativos a $\mathbf{0 , 0 0 1 \%}$.

orgânico e capacidade máxima de adsorção de $\mathrm{P}$ (Quadro 3). Resultados similares foram apresentados por Mendoza (1986); López Camelo (1989) e Singh \& Gilkes (1991). Por outro lado, López Camelo et al. (1986), trabalhando com solos semelhantes, comprovaram que Xm diminuiu, quando foram incorporados ácidos húmicos, e aumentou, quando parte do carbono orgânico do sol o foi eliminado. Tais resultados refletem que o papel da matéria orgânica em relaçãoà adsorção de $P$ não tem tendência definida.

\section{CONCLUSÕES}

1. Os sol os de E ntreRíos caracterizam-se por terem fator capacidade de fósforo de baixo a muito baixo.

2. A capacidade máxima de adsor ção de $P$ do solos mostrou-se correlacionada com teor deargila, mas não com o de carbono orgânico.

3. Um índice simples, o fósforo remanescente, permite classificar os solos quanto ao seu fator capacidade e estimar sua capacidade máxima de adsorção de P. 


\section{AGRADECIME NTOS}

A C.J . Vesco, pela identificação e classificação das amostras dos solos. Este trabalho foi financiado por SCYTFRH-UNER.

\section{LITE RATURA CITADA}

ALVAREZ V., V.H. Efecto de los factores cantidad, intensidad y capacidad amortiguadora de fosfato en la evaluación del fósforo disponible, de suel os derivados de cenizas vol cánicas de la Mesata Tarasca. Chapingo, México, Colegio de Postgraduados, 1982. 300p. (Tese de Doutorado)

ALVAREZ V., V.H. \& FONSECA, D.M. Definição de doses de fósforo para determinação da capacidade máxima deadsorção de fosfatos e para ensaios em casa de vegetação. $\mathrm{R}$. Bras. Ci. Solo, 14:49-55, 1990.

BAHIA FILHO, A.F. Fósforo em Latossolos do estado de Minas Gerais: Intensidade, capacidade tampão e quantidade de fósforo, fósforo "disponível" e crescimento vegetal. Viçosa, Universidade Federal de Viçosa, 1974. 69p. (Tese de Mestrado)

BOLAÑO, A.A. Determinación de la capacidad reguladora y concentración ajustada de fósforo en suelos de la región Pampeana. Ci. Suelo, 2:99-106, 1984.

BUSCHIAZZO, D.E.; HEVIA, G.G. \& HEPPER, E.N. Variación de la adsorción de fósforo y parámetros edáficos en una climosecuencia de suelos de la región Semiárida Pampena Central. Ci. Suelo, 8:119-126, 1990.

CAJ USTE, L.J .; ALVAREZ V., V.H. \& LAIRD, R.J . Evaluation of chemical extractants for assessing phosphate availability in vol canic ash soils. Comm. Soil Sci. Plant Anal., 23:2189-2206, 1992.

DEAR, B.S.; HELYAR, K.R.; MULLER, W.J . \& LOVELAND, B. The $P$ fertilizer requirements of subterranean clover, and the soil P status, sorption and buffering capacities from two P analyses. Aust. J. Soil Res., 30:27-44, 1992.

FREIRE, F.M.; NOVAIS, R.F.; BRAGA, J .M.; FRANCA, G.E.; SANTOS, H.L. \& SANTOS, P.R. Adubação fosfatada para a cultura da soja (Glycinemax) baseada no fósforo disponível e no fator "capacidade". R. Bras. Ci. Solo, 3:105-111, 1979.

HEPPER, E.N.; HEVIA, G.G. \& BUSCHIAZZO D.E. Adsorción de fósforo y su relación con los parámetros edáficos en una climotoposecuencia de la región semiárida pampeana central Argentina. Agrochimica, 39:26-32, 1995.

HOLFORD, I.C.R. \& MATTINGLY, G.E. G. Phosphateadsorption and availability. Plant Soil, 44:377-386, 1976.

LÓPEZ CAMELO, L.G.; HEREDIA, O.S.; NERVI, A. \& SESE, Z. $M$. Adsorción de fósforo en algunos suelos argentinos. I . Condiciones experimentales e isotermas de adsorción. R. Fac. Agron., 5:165-174, 1984.
LÓPEZ CAMELO, L.G.; HEREDIA，O.S. \& SESE, Z.M. Evaluación preliminar dela influencia dela materia orgánica sobre la adsorción de fósforo en algunos suelos argentinos. Ci. Suelo, 2:125-131, 1986.

LÓPEZ CAMELO, L.G. \& HEREDIA O.S. Indices de sorción de fosfóro en algunos molisoles de la pradera pampeana. R. Fac. Agron., 9:145-154, 1989.

MENDOZA, R.E. I sotermas de adsorción de fósforo en suelos argentinos. II - Aplicabilidad de las isotermas e interrelación de sus parámetros con variábles de suel o y planta. Ci. Suelo, 2:117-124, 1986.

MOUGHLI, L.; WESTFALL, D.G. \& ALI BOUKHIAL. Use of adsorption and buffer capacity in soil testing for phosphorus. Comm. Soil Sci. Plant Anal., 24:1959-1974, 1993.

MULJ ADI, D.; POSNER, A.M. \& QUIRK, J .P. The mechanism of phosphate adsorption by kaolinite, gibbsite, and pseudoboehmite. Part 1. The isotherms and effect of $\mathrm{pH}$ on adsorption. J. Soil Sci., 17:212-229, 1966.

MUNIZ, A.S.; NOVAIS, R.F.; BARROS, N.F. \& NEVES, J.C.L. Nível crítico de fósforo na parte aérea de soja como variável do fator de capacidade de fósforo no solo. R. Bras. Ci. Solo, 9:2337-243, 1985.

OLSEN, S.R. \& WATANABE, F.S. A method to determine a phosphorus adsorption maximum of soils as measured by the Langmuir I sotherm. Proc. Soil Sci. Soc. Am., 21:144-149, 1957.

OZANNE, P.G. \& SHAW, T.C. Advantages of the recently devel oped phosphate sorption test over the older extractant methods for soil phosphate. In: INTERNATIONAL CONGRESS OF SOIL SCIENCE, 9., Adelaide, Australia, 1968. Transactions. Adelaide, 1968. v.2. p.273-280.

QUINTERO, C.E.; BOSCHETTI, N.G. \& BENAVIDEZ, R.A. Estimación de la capacidad máxima de adsoción de fosfatos en suelos deEntre Ríos (Argentina). Ci. Suelo, 14:79-82, 1996.

RON, M.M.; BUSSETTI, S.G. \& LOEWY, T. Uso de un índice de sorción como complemento del fósforo extraíble para la fertilización de trigo. Ci. Suelo, 13:35-37, 1995.

SILVA, J .T.A. \& BRAGA, J .M. Influência das características físicas e químicas sobre o fator capacidade-tampão de fósforo em latossolos do estado de Minas Gerais. R. Ceres, 41:575-583, 1993.

SILVA, J .T.A. Relações entre características do sol o que refletem a capacidade-tampão de fósforo e o nível crítico para sorgo, em dois latossolos de Minas Gerais, em casa de vegetação. Viçosa, U niversidade Federal de Viçosa, 1990, 87p. (Tese de Mestrado)

SINGH, B. \& GILKES, R.J . Phosphorus sorption in relation to soil properties for the major soil types of south-western Australia. Aust. J . Soil Res., 29:603-618, 1991. 